\title{
Merkel Cell Carcinoma: Update and Review
}

\author{
Timothy S. Wang, MD," Patrick J. Byrne, MD, FACS, ${ }^{\dagger}$ Lisa K. Jacobs, MD, ${ }^{\ddagger}$ \\ and Janis M. Taube, MD ${ }^{\S}$
}

\begin{abstract}
Merkel cell carcinoma (MCC) is a rare, aggressive, and often fatal cutaneous malignancy that is not usually suspected at the time of biopsy. Because of its increasing incidence and the discovery of a possible viral association, interest in MCC has escalated. Recent effort has broadened our breadth of knowledge regarding $\mathrm{MCC}$ and developed instruments to improve data collection and future study. This article provides an update on current thinking about the Merkel cell and MCC.
\end{abstract}

Semin Cutan Med Surg 30:48-56 @ 2011 Elsevier Inc. All rights reserved.

$\mathrm{M}$ erkel cell carcinoma (MCC) is a rare, aggressive, and often fatal cutaneous malignancy. It usually presents as a banal-appearing lesion and the diagnosis is rarely suspected at the time of biopsy. Because of increasing incidence and the discovery of a possible viral association, interest in MCC has escalated rapidly.

From 1986 to 2001, the incidence of MCC in the United States has tripled, and approximately 1500 new cases are diagnosed each year. ${ }^{1,2}$ MCC occurs most frequently among elderly white patients and perhaps slightly more commonly in men. MCCs tend to occur on sun-exposed areas, with nearly $80 \%$ presenting on the head, neck, and extremities. ${ }^{3}$ Immunosuppression increases the relative risk of MCC with an approximately 13-fold increase in patients with HIV and a 10-fold increase in solid-organ transplant patients. ${ }^{4,5}$ Patients with chronic lymphocytic leukemia (CLL) have an increased risk of MCC as well. ${ }^{6}$ MCC is particularly aggressive with a relative mortality of approximately $30 \%$ at 2 years after diagnosis and $50 \%$ at 5 years after diagnosis. ${ }^{7}$ Many patients present with metastatic disease, and there is a high risk of local, regional, and distant recurrence despite treatment.

Recent research has broadened our breadth of knowledge on the subject of MCC. This article provides an update on

*Division of Cutaneous Surgery and Oncology, Department of Dermatology, The Johns Hopkins University School of Medicine, Baltimore, MD

$\dagger$ Division of Facial Plastic and Reconstructive Surgery, Departments of Otolaryngology Head and Neck Surgery and Dermatology, Johns Hopkins University School of Medicine, Baltimore, MD

¥Department of Surgery, Johns Hopkins University School of Medicine, Baltimore, MD

$\S$ Departments of Dermatology and Pathology, The Johns Hopkins University School of Medicine, Baltimore, MD.

Address reprint requests to Timothy S. Wang, MD, Johns Hopkins University School of Medicine, 601 North Caroline Street, Baltimore, MD 21287-0900. E-mail: twang49@jhmi.edu current thinking, including novel insights into the Merkel cell; a review of the 2010 National Comprehensive Cancer Network (NCCN) therapeutic guidelines and new American Joint Committee on Cancer (AJCC) staging system; recommendations for pathologic reporting and new diagnostic codes; and the recently described Merkel cell polyoma virus $(\mathrm{MCPyV})$.

\section{History}

Merkel cells (MCs) were first described by Friedrich Merkel in 1875 as clear cells associated with nerve fibers. ${ }^{8}$ Merkel assumed that MCs were involved in sensation, and called them tastzellen or touch cells. MCC was first described by Toker in $1972 .{ }^{9}$ Termed "trabecular cell" carcinoma, reports of its pathogenesis, course, and treatment were relatively sparse and mostly limited to case reports or small series. Efforts to advance knowledge in the disease were confounded by its rarity, lack of reliable specific markers for diagnosis, and varied staging systems. Because of their similar histologic characteristics, MCC is generally believed to derive from cutaneous MCs or a common precursor.

\section{Clinical Presentation}

The primary lesion of MCC is distinguished by its absence of distinctive clinical characteristics. Rarely suspected at the time of biopsy, the clinical differential diagnosis includes more common lesions, such as basal cell carcinoma (BCC), epidermoid cyst, or even amelanotic melanoma. MCC often presents as a rapidly growing, asymptomatic, reddish-blue dermal papule or nodule that develops over the course of weeks to months (Fig. 1). The mnemonic AEIOU has been used to describe its clinical appearance and demographic characteristics: asymptomatic, expanding rapidly, immune 


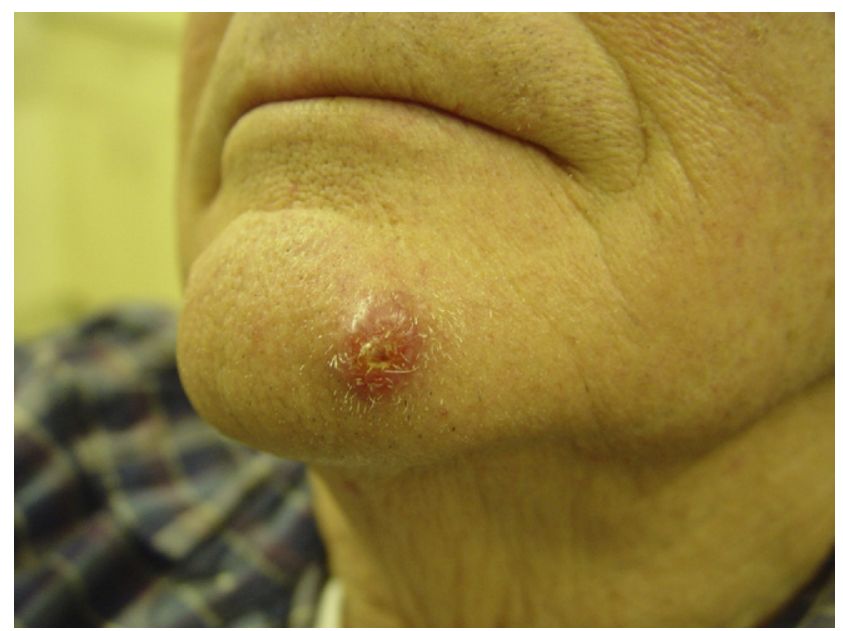

Figure 1 Primary MCC, chin.

suppression, older than 50 years, ultraviolet-exposed/fair skin. ${ }^{10}$ However, most of these tumors are likely diagnosed through a combination of vigilance, a low threshold for biopsy, and microscopic evaluation, rather than by clinical findings.

\section{Histology}

\section{The Merkel Cell}

MCs are clear oval cells measuring 10-15 $\mu \mathrm{m}$ in diameter. They are found most commonly in the basal layer of the epidermis in specialized groupings called haarscheiben, in the outer root sheath of hair follicles and in the skin of the palms and soles. They are also found in oral mucosa and are rarely distributed singly in interfollicular skin. ${ }^{11}$ MCs in haarscheiben, palms and soles, and interfollicular skin are usually associated with a sensory axon forming a Merkel cell-neurite complex. However, in hair follicles and mucosa, MCs are often not associated with a nerve. ${ }^{11}$

Haarscheiben are specialized structures of epidermis containing multiple MCs and associated nerve fibers. They were originally described in animals, where they are associated with whisker and sinus hairs and thought to signal directional movement. In humans, however, they are not consistently associated with hairs and are more commonly found on the neck and abdomen. In animals, they are sometimes visible to the naked eye on depilated skin, but in humans they are not. Other names for haarscheiben include touch domes, hair disks and tastflecke. ${ }^{12}$

In hair follicles MCs are found in 2 distinct bands, one near the bulge and the other close to the skin surface in the upper infundibulum. In the palms and soles, they are found at the base of epidermal ridges and are especially numerous on the fingertips. On the basis of studies of structural proteins, distinct populations of MCs have been shown to exist at various locations in the body. ${ }^{13}$

Whether MCs arise from epidermal or neural crest progenitors has been a matter of controversy for many years. Recent evidence suggests that MCs arise from epidermal progenitors during embryonic development; in adults, they mature and are then replaced from an epidermal stem cell source-not from the proliferation of differentiated MCs. ${ }^{14}$

MCs have long been considered important in the response to touch. Nerve fibers associated with MCs are slowly-adapting type 1 fibers, important in light touch and discrimination of fine detail. Mice genetically deficient in MCs demonstrated an absence of slowly-adapting type 1 mechanoreceptor responses, lending support to the Merkel cell's presumed role in light touch. ${ }^{15}$

MCs are difficult to see on light microscopy. Merkel himself used special techniques to visualize these cells and even today, without immunohistochemical stains, they are nearly impossible to detect. Their morphologic description has been gleaned principally by use of electron microscopy. Their hallmarks include lobulated nuclei; a loose cytoskeletal network of intermediate filaments; electron-dense (dense-core) membrane-bound cytoplasmic granules; and spine-like microvilli protruding from the cell surface into invaginations in surrounding keratinocytes. These projections may play a role in "connecting" the Merkel cell to its surrounding cells, thereby enhancing mechanical sensation. ${ }^{13}$ In different tissues and even within similar tissues, their spine-like protrusions are highly variable in length.

Immunohistochemically, MCs demonstrate both epithelial and neuroendocrine markers. The loosely arranged intermediate filaments stain positively for low molecular weight cytokeratins (CK) 8, 18, 19, and 20. ${ }^{16} \mathrm{CK} 20$ in particular has been shown to be a highly specific marker for MCs in normal skin. ${ }^{17}$ In normal MCs, CK20 stains in a diffuse manner, rather than in the paranuclear pattern seen in MCC. The dense-core granules stain positively for the neuroendocrine markers chromgranin A, neuron-specific enolase, and synaptophysin, and immunohistochemistry for these markers may be useful as diagnostic adjuncts. Vasoactive intestinal polypeptide, serotonin and substance $\mathrm{P}$ show variable positivity.

\section{Merkel Cell Carcinoma}

MCCs are dermally based tumors composed of small uniform round blue cells arranged in anastomosing cords, bands and clusters. Their cells commonly possess ill-defined, scanty cytoplasm, and round vesicular nuclei with "salt and pepper" chromatin and frequent mitotic figures (Fig. 2A). Up to 10\% of MCCs contain pagetoid intraepidermal involvement and apoptotic bodies are often seen. ${ }^{18}$ MCCs sometimes contain areas of squamotization and can occur in combination with other epithelial tumors. Nearly $40 \%$ are associated with adjacent or overlying Bowen's disease or squamous cell carcinoma. ${ }^{19}$ Less frequently, MCCs are found in association with BCC or eccrine tumors.

Many terms have been used to describe the architecture of MCCs, including the trabecular pattern as originally described by Toker, which is thought to account for approximately $10 \%$ of MCCs. ${ }^{20}$ Other recognized patterns include organoid, ribbon-like, diffuse, intermediate, and mixed and small types. Whether these subtypes prove to be of prognostic significance is yet undetermined. The most recent recom- 


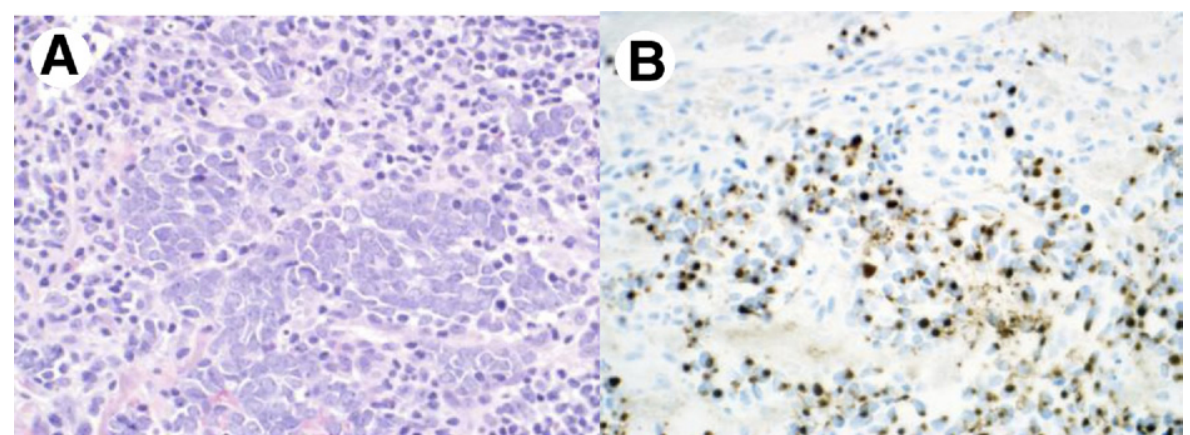

H\&E (original magnification 200X) CK20 (original magnification 200X)

Figure 2 Microscopic appearance of MCC. (A) H\&E (original magnification, 200X) B. CK20 (original magnification, $200 \times)$.

mendations by the College of American Pathologists suggest that a growth pattern of nodular or infiltrative be specifically recorded.

The histopathologic differential diagnosis for MCC includes other small round blue cell tumors, such as metastatic small cell carcinoma of the lung, small cell cutaneous lymphoma, melanoma, Ewing's sarcoma, neuroblastoma, rhabdomyosarcoma, and BCC.

Immunohistochemistry is often used to secure the diagnosis of MCC and, when used appropriately, is usually definitive. CK20 is the predominant tool used by pathologists, and stains approximately $80 \%-90 \%$ of all MCCs in a distinctive paranuclear dot-like pattern..$^{21}$ (The terms peri- and paranuclear have been used throughout the literature to describe the same phenomenon.) The characteristic paranuclear staining is due to clumping of intermediate filaments (Fig. 2B)

Neurofilament immunostains are also positive in a paranuclear dot pattern in up to $95 \%$ of MCCs (greater than CK20) and may be useful as a primary stain and/or in CK20-negative MCCs. ${ }^{22}$ Positive thyroid transcription factor- 1 staining is useful in differentiating small cell carcinoma of the lung from MCC. As in normal MCs, MCCs frequently stain positively with neuroendocrine markers, ie, chromgranin A, neuronspecific enolase, and synaptophysin. CM2B4 is an antibody that recognizes the Large $\mathrm{T}$ (LT) antigen of the MCPyV and has been demonstrated in approximately $70 \%$ of MCC. ${ }^{5} \mathrm{Ta}$ ble 1 lists the immunostains that are generally useful for making the diagnosis of MCC.

Table 1 Immunostains for MCC

\begin{tabular}{|c|c|c|c|c|}
\hline Stain & MCC & $\begin{array}{l}\text { Small Cell } \\
\text { Lung Cancer }\end{array}$ & Lymphoma & Melanoma \\
\hline CK 20 & + & - & - & - \\
\hline CK 7 & - & + & - & - \\
\hline TTF-1 & - & + & - & - \\
\hline LCA & - & - & + & - \\
\hline $\mathrm{S} 100$ & - & - & - & + \\
\hline
\end{tabular}

CK, cytokeratin; TTF-1, thyroid transcription factor-1; LCA, leukocyte common antigen.

\section{New Pathology Reporting Recommendations}

The College of American Pathologists develops protocols to assist pathologists in reporting relevant pathologic information to clinicians. In 2010, they released recommendations for reporting MCC of the skin. ${ }^{23}$ The protocol consists of a checklist of required and suggested elements for the pathologic reporting of cutaneous MCC and not only includes elements found in the most recent AJCC staging system but also suggests reporting additional characteristics that may prove to be valuable. Thus, in addition to required elements of tumor site, size, margins, etc., suggested elements include tumor thickness, lymph-vascular invasion, mitotic index/ $\mathrm{mm}^{2}$, tumor-infiltrating lymphocytes, tumor growth pattern and pathologic staging (Table 2). Explanatory notes and diagrams are included to assist the pathologist and clinician in understanding and interpreting these characteristics. Clini-

\section{Table 2 Checklist for Reporting MCC}

\author{
Required elements \\ Type of procedure (excision, re-excision, SLN etc) \\ Macroscopic tumor (presence or absence) \\ Tumor site \\ Tumor size (mm) \\ Margins (peripheral and deep) \\ Lymphovascular invasion (presence or absence) \\ Invasion of bone, muscle, fascia or cartilage \\ Lymph nodes (total number and number positive, \\ presence or absence of macroscopic tumor) \\ Pathologic staging ( $\mathrm{pTNM}$ ) \\ Suggested elements \\ Tumor thickness (mm) \\ Mitotic index (per $\mathrm{mm}^{2}$ ) \\ Tumor-Infiltrating lymphocytes (presence: brisk or \\ nonbrisk or absence) \\ Tumor growth pattern (nodular or infiltrative) \\ Presence of second malignancy \\ Lymph nodes (size of largest metastatic focus and \\ presence or absence of extra-nodal extension)
}

Adapted from Rao et al. ${ }^{23}$ 
Table 3 TNM Criteria and Stage Groupings of New American Joint Committee on Cancer Staging System for Merkel Cell Carcinoma

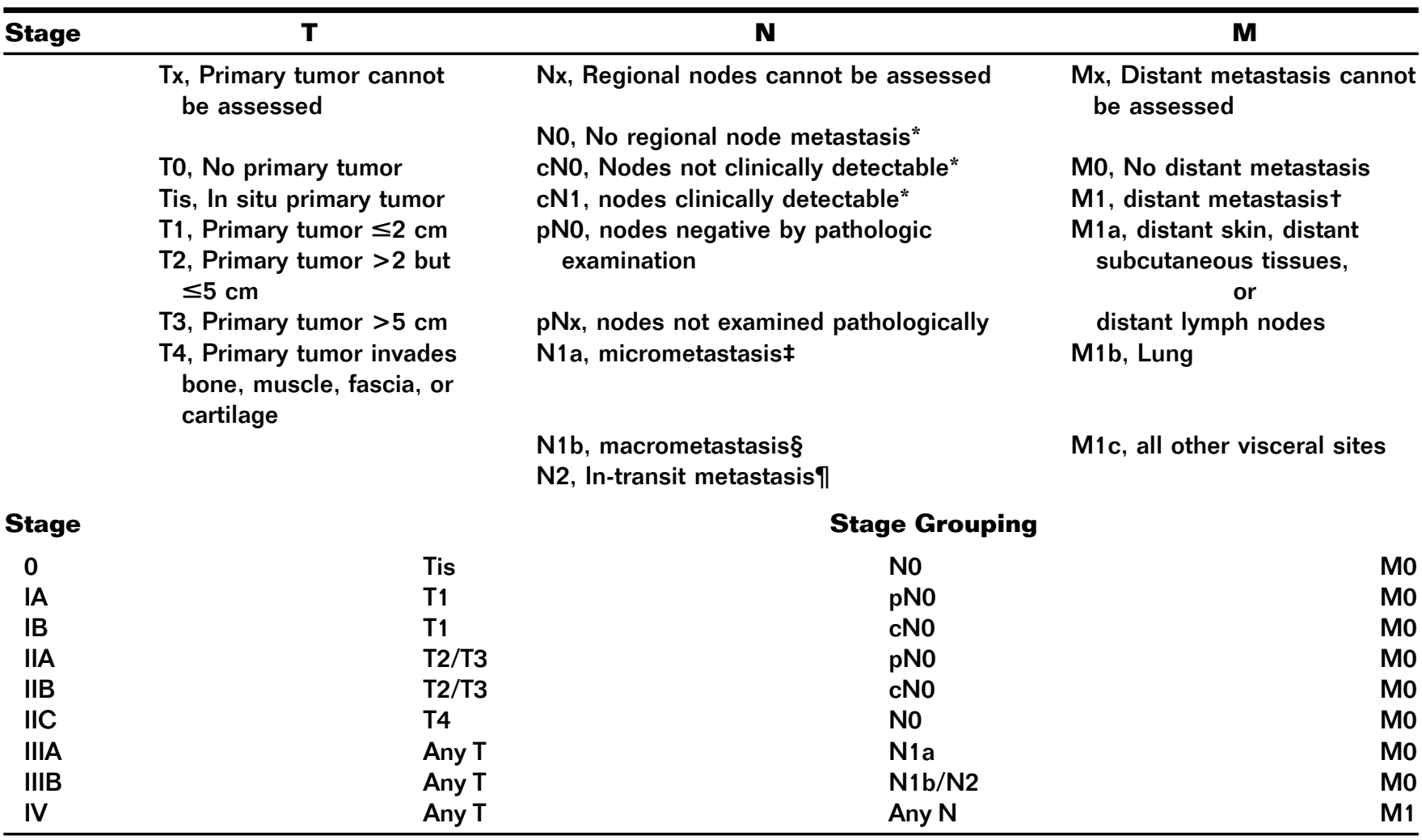

From Lemos et al. ${ }^{7}$

*"NO" denotes negative nodes by clinical, pathologic, or both types of examination. Clinical detection of nodal disease may be via inspection, palpation, and/or imaging; cNO is used only for patients who did not undergo pathologic node staging.

tBecause there are no data to suggest significant effect of $M$ categories on survival in Merkel cell carcinoma, M1a-c are included in same stage grouping.

¥Micrometastases are diagnosed after sentinel or elective lymphadenectomy.

§Macrometastases are defined as clinically detectable nodal metastases confirmed pathologically by biopsy or therapeutic lympadenectomy.

ПIn-transit metastasis is tumor distinct from primary lesion and located either: (1) between primary lesion and draining regional lymph nodes; or (2) distal to primary lesion.

cians should familiarize themselves with these recommendations and request them from pathologists reporting MCC.

\section{Staging}

In the recent past, 5 staging systems have been proposed for MCC. ${ }^{7}$ MCC is a rare malignancy and these systems were often based on single-institution experience with relatively few patients and short follow-up. In 2010, the AJCC released its first-ever consensus staging system for MCC. Their proposed tumor-nodes-metastases (TNM) criteria and stage groupings are based on analysis of national Cancer DataBase data from more than 4000 MCC patients with at least 5 years' follow-up and thus represent a significant advancement in the study of MCC. The proposed system adopts many definitions used in the classification of melanoma and other malignancies and maintains the architecture of other AJCC staging systems. It will likely lead to better standardization of patient data and prognostic information and aid the clinician in counseling patients with MCC (Table 3).

The following is a brief review of the AJCC findings which form the basis for their proposed TNM criteria and stage groupings. As noted in previous studies, significant differences have been found between patients presenting with local, regional and distant metastatic disease (Figs 3 and 4).

\section{Primary Tumor (T Category)}

The characteristics used in defining the $\mathrm{T}$ category are the size of the primary tumor and/or invasion of deep tissues, such as bone, muscle, fascia or cartilage. Consistent with previous studies, the size of the primary tumor was found to be an important factor in survival. In patients with local disease only, primary tumor size of $<2 \mathrm{~cm}$ appears to be a natural prognostic break-point; patients with primary tumors $\leq 2 \mathrm{~cm}$ in diameter (T1) fared better than those with tumors $>2 \mathrm{~cm}$ (T2 and T3). No difference in survival was found in patients with primary tumors $>2 \mathrm{~cm}$ in diameter, and the T2 and T3 categories were both therefore included in the same stage grouping, II. The T4 category was added specifically to represent deeply invasive tumors, ie, those invading bone, muscle, fascia or cartilage, as in other AJCC staging systems. For all patients (presenting with local, regional or distant disease), the size of the primary tumor had a significant impact 


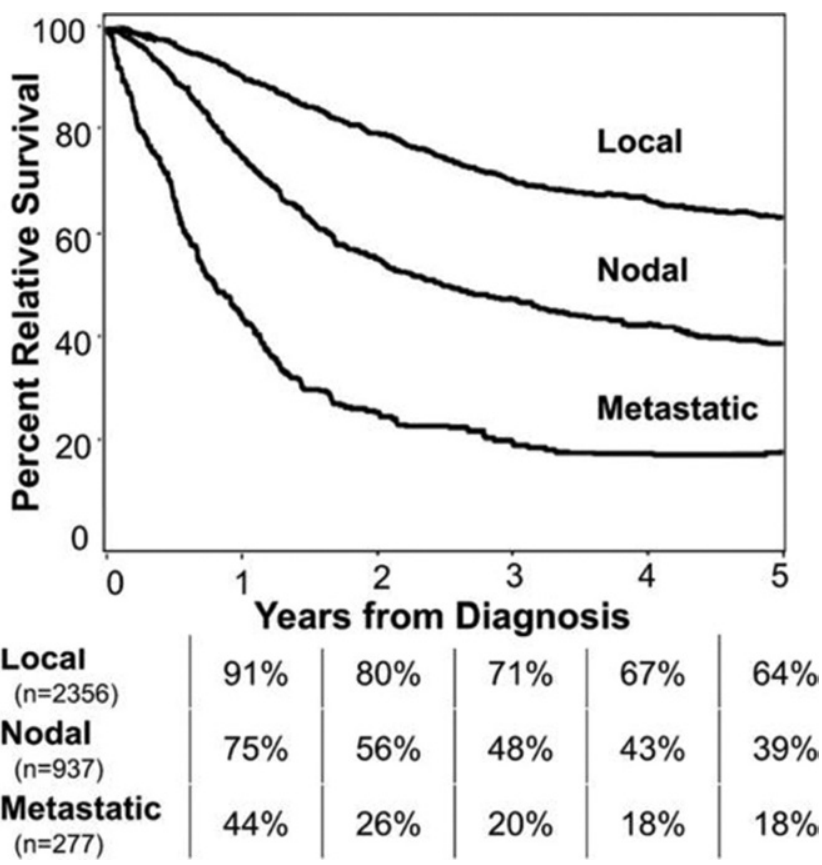

Figure 3 Relative survival by extent of MCC. From Lemos et al. ${ }^{7}$

on survival when the primary tumor was $\leq 4 \mathrm{~cm}$. No impact on survival was noted for tumors $>4 \mathrm{~cm}$.

\section{Regional Lymph Nodes (N Category)}

The N classification is determined by whether the patient's regional lymph nodes have been clinically or pathologically staged and in patients with nodal disease whether their dis- ease is palpable (macroscopic) or not (microscopic). The N2 category was added solely to denote in-transit disease.

Upon analysis of the data, patients categorized as node negative by clinical examination only were found to do more poorly than those who were classified as node negative after histopathologic examination of clinically negative lymph nodes. Thus, clinically node-negative (cNO) and pathologically node-negative ( $\mathrm{pNO}$ ) criteria have been incorporated into the proposed staging system. The distinction between clinical and pathologic staging is important, as fully one-third of patients presenting with local disease harbor occult metastases. ${ }^{24}$ Pathologic staging of clinically negative lymph nodes (such as by sentinel lymph node [SLN] biopsy) has been shown to improve prognostic accuracy. ${ }^{7}$

In patients with confirmed nodal disease, those with clinically palpable (macroscopic) nodal disease (N1b) did worse than those with microscopic (clinically negative but found to be positive on pathologic examination, such as by SLN biopsy) disease (Nla). Thus, Nla and Nlb are categorized in sub-stages IIIA and IIIB, respectively. In-transit cutaneous disease (either between the primary site and the primary nodal basin or distal to the primary site) is classified as N2.

\section{Distant Metastasis (M Category)}

The prognosis for patients presenting with stage IV disease is grave, with an approximately $44 \% 1$-year survival. ${ }^{7}$ The M classification is divided into the absence or presence of distant metastatic disease ( $\mathrm{M} 0$ and $\mathrm{Ml}$, respectively). Although there is no evidence of survival differences based on the site of metastasis, the $\mathrm{Ml}$ category is subdivided into Mla—distant
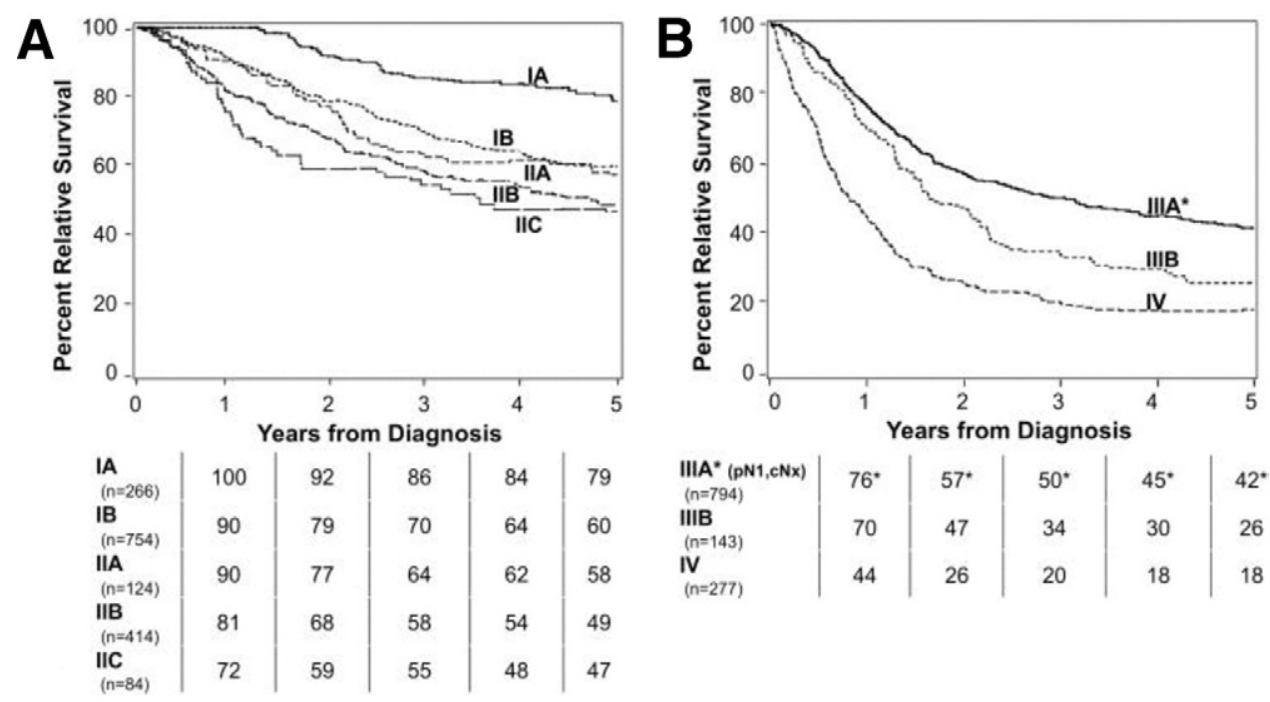

Figure 4 Relative survival for MCC by stage at presentation in 2856 patients. Relative survival for MCC by stage at presentation in 2856 patients. Sufficient local, nodal, and distant data were available for stage classification of 2856 patients with MCC as summarized in Fig. 2. Cases presenting with local (A) or regional nodal/distant metastatic (B) disease are shown by substages with annual percent relative survival below each panel. Stages are as indicated directly on survival curves except for stage IIIA (microscopic node positive, clinical node negative), which could not be derived using National Cancer DataBase as described in the "Discussion" section. Curve IIIA* represents pathologically nodepositive patients whose clinical node status was unknown ( $p N 1, c N x)$. It is anticipated that patients at true stage IIIA with known clinically negative node status ( $\mathrm{pN} 1, \mathrm{cN} 0)$ may have better survival than line marked IIIA* (pN1, cNx). From Lemos et al. ${ }^{7}$ 
Table 4 New ICD Codes for Merkel Cell Carcinoma

- 209.31-Merkel cell carcinoma of the face

- 209.32 - . . Scalp and neck

- 209.33 - . . U Upper limb

- 209.34 - . . L Lower limb

- 209.35 - . . Trunk

- 209.36 - . . . Other sites.

- 209.75-Merkel cell carcinoma, unknown primary site, nodal presentation, secondary (any site), visceral metastatic presentation.

V10.9 (unspecified personal history of malignant neoplasm) expanded to:

- V10.90-Personal history of unspecified type of malignant neoplasm

- V10.91—Personal history of malignant neuroendocrine tumor.

From Beebe et al. ${ }^{26}$

skin, subcutaneous tissue or distant lymph node involvement, Mlb_lung, and Mlc_all other visceral sites. These subdivisions were devised to mirror the staging system used in melanoma, where survival differences between these sites have been demonstrated. ${ }^{25}$

\section{Stage Groupings}

Patients with local disease only are categorized as stage I or II, stage I for patients with primary tumors $\leq 2 \mathrm{~cm}$ and stage II for those $>2 \mathrm{~cm}$. Subdivisions of stages I and II are based on size of the primary tumor and whether the absence of nodal disease is based on clinical findings or pathologic evaluation of a clinically negative lymph node (such as by SLN biopsy). Stage IIC was added to distinguish node negative patients (either clinically or pathologically) with tumors involving bone, muscle, fascia or cartilage (deeply invasive). Patients with regional disease-stage III, are subdivided into those with micrometastases (discovered on pathologic examination only) vs. macrometastases (determined either clinically or on imaging) or in-transit disease. There are no subdivisions of stage IV disease.

\section{New Diagnostic Codes}

In 2009, 7 new International Classification of Diseases (ICD) codes for MCC were developed and adopted (Table 4). ${ }^{26}$ The new MCC codes describe primary cutaneous MCCs at specific sites (209.3x) and metastatic MCC (209.75) with unknown primary site, nodal presentation, secondary (any site), or visceral metastatic presentation.

Before the adoption of these codes, MCC was coded in the ICD system as 173.x, which includes BCC and SCC, tumors that rarely require aggressive therapy. As a result, for patients with MCC classified as 173.x, obtaining insurance approval for treatment was sometimes difficult.

For follow-up after treatment, the V10.9 code: "Unspecified personal history of malignant neoplasm" has been expanded to include V10.91: "Personal history of malignant neuroendocrine tumor." These new codes should facilitate more accurate classification of patients with MCC for research and insurance purposes.

\section{Treatment}

The main components of MCC therapy are surgery and radiotherapy. MCC has been demonstrated to be radiosensitive and radiotherapy is now used mainly as an adjuvant to surgery. Ultimately, aggressive surgery may prove to be overused and radiotherapy underused in MCC. At present, much controversy exists and the specific roles of these therapeutic modalities are still being elucidated. The National Comprehensive Cancer Network annually updates consensus recommendations for the treatment of MCC. The following is a summary of their recommendations and discussions with panel members and experts in the field. ${ }^{27}$ A multidisciplinary approach to treatment of MCC is vital to optimize outcomes. ${ }^{28}$

\section{Surgery}

The mainstay of therapy for patients newly diagnosed with primary MCC remains surgical. Current recommendations are based on the clinical size of the primary tumor, and call for tumor excision with $1 \mathrm{~cm}$ margins for tumors $<2 \mathrm{~cm}$ in size and $2 \mathrm{~cm}$ margins for those $>2 \mathrm{~cm}$ in size. ${ }^{27}$ Radiotherapy has been used as monotherapy for primary tumors with reported success, but until more data become available, surgery remains the mainstay of therapy for primary MCC tumors. ${ }^{29,30}$

Because nearly one-third of clinically node-negative patients harbor microscopic nodal disease, SLN biopsy is currently recommended for all untreated primary tumors at the time of wide local excision. ${ }^{24}$ SLN biopsy has been shown to be important in the staging and prognosis of MCC, and SLN status is included in the most recent AJCC staging guidelines. ${ }^{7}$ SLN biopsies should be examined by both hematoxylin and eosin (H\&E) and immunoperoxidase staining, including CK20.

If sentinel nodes are positive, completion lymph node dissection (CLND) of the nodal basin followed by radiotherapy of the basin is recommended. In cases where SLN positivity is found on immunostaining but not $H \& E$ staining of the lymph node, radiotherapy without CLND may be considered as sole regional therapy.

SLN biopsy on the head and neck can be technically challenging but may have prognostic value and is therefore recommended. As in other cancers, the predictive value of SLN biopsy relies on intact lymphatic drainage patterns at the site of the primary tumor, and SLN biopsy should be performed at the time of wide local excision. Although it has been suggested that SLN may not be indicated for primary MCC $<1$ $\mathrm{cm}$, SLN positivity has been shown sometimes to be independent of primary tumor size. ${ }^{31,32}$

\section{Radiation Therapy}

MCC is considered a radiosensitive neoplasm and radiotherapy plays an important role in its management. Although its 
role in MCC treatment may ultimately increase, radiotherapy is currently used as an adjunct to surgery, and as primary therapy only in inoperable cases or when the patient refuses surgery. Areas for consideration of radiotherapy include the primary site, the draining nodal basin and intervening intransit lymphatics.

After wide local excision, adjuvant radiotherapy to the primary site is recommended in nearly all cases of MCC. Radiotherapy to the primary tumor site has been shown to decrease the incidence of local recurrence 3.7-fold. ${ }^{33}$ Radiotherapy to the primary site may be considered optional in patients deemed at lowest risk for local recurrence, including immunocompetent patients whose primary tumor measured $<1$ $\mathrm{cm}$ and possessed no adverse histologic features, whose wide local excisions showed unequivocally clear margins, and whose SLN biopsy was negative by both H\&E and immunohistochemical staining.

Recommendation for adjuvant radiation to the draining nodal basin is dictated by SLN status and confidence in the predictive value of the SLN biopsy. There is evidence that surgery plus adjuvant radiotherapy to the regional nodal basin significantly decreases the rate of regional recurrence and improves survival. ${ }^{33,34}$ If the SLN is positive, radiotherapy after CLND to the nodal basin is recommended. Patients at greatest risk for regional recurrence include those with macroscopic nodal disease, multiple node involvement or extracapsular extension. Although the use of radiotherapy alone for regionally metastatic MCC has been described, thoughtful commentary highlights the difficulties involved with study design in this patient population. ${ }^{35,36}$

In cases in which SLN biopsy was not performed or when the predictive value of the SLN biopsy may be questioned (eg, altered draining lymphatics or lack of immunohistochemical staining), radiotherapy to the draining basin should be considered. Because of the complexity of SLN biopsy in the head and neck, adjuvant radiation should be considered even in the setting of a negative SLN in this region. For lesions on the trunk or extremities where SLN biopsy is felt to be reliable, radiation may not be indicated when the SLN is negative on both H\&E and immunohistochemistry.

In light of MCC's relative radiosensitivity, adjuvant radiotherapy is generally recommended in all patients except for those at lowest risk. Risk assessment is based on patient factors (such as immune status, overall health), tumor and nodal characteristics (including size of the primary tumor, lymphovascular invasion, depth of invasion, and extracapsular extension) and technical aspects of therapy (altered lymphatics, challenging location, compromised margins, lack of SLN biopsy or immunohistochemical staining). The morbidity of adjuvant radiotherapy, especially after CLND, must always be weighed against the risk of recurrence.

\section{Chemotherapy}

The role of chemotherapy in the treatment of MCC remains unclear. Although a majority of patients with locally advanced or distant disease may initially respond to treatment with chemotherapy, no survival benefit has been demon- strated in these patients. ${ }^{37}$ The most commonly used agents combine platinum containing agents with etoposide and are associated with significant morbidity and, in some cases, mortality. ${ }^{38}$ Chemotherapy is therefore currently considered as palliative treatment in cases of disseminated disease, but not as an adjuvant or primary therapy unless clinically warranted.

\section{Merkel Cell Polyoma Virus}

In 2008, Feng et al characterized a novel polyomavirus, the $\mathrm{MCPyV}$, and inferred an association between it and the pathogenesis of MCC. This seminal work has sparked great interest in MCC and has opened a new pathway in the study of viral tumorigenesis. ${ }^{39,40}$

Feng's group detected MCPyV DNA in $80 \%$ of MCC tumors but in only $16 \%$ of normal tissue samples. Viral DNA was found to be clonally integrated within an individual tumor's genome, suggesting that viral infection and integration occurred before clonal expansion of the tumor cells. In one patient, an identical integration pattern was found in the primary tumor and a metastatic lymph node, supporting the notion that viral integration and clonal expansion preceded metastasis. In different MCC tumors, MCPyV DNA sequences were found to be integrated at different sites within the genome.

Further work by this group described mutations within the $\mathrm{LT}$ antigen coding region of the MCPyV. These mutations were found in MCPyV positive MCC tissue samples but not in MCPyV-positive control (non-MCC) samples, allowing at least an association to be drawn between these mutations and MCC tumors. The MCPyV found in control samples was thus felt to be epigenomic, wild-type MCPyV. The authors propose that MCPyV-associated transformation of MCs into MCC is a 2-step process: (1) integration of MCPyV DNA into the genome and (2) LT antigen mutation.

Polyoma viruses are small double-stranded DNA viruses. Although known to be capable of producing multiple tumors in animal models (hence the term poly + oma), none has been proven to cause tumors in humans. To date, 14 have been identified and 6 (including the MCPyV) infect humans. They are $\mathrm{BK}, \mathrm{JC}, \mathrm{KI}, \mathrm{WU}$, and $\mathrm{MCPy}$ viruses and the very recently discovered TS virus. All polyoma viruses encode T antigen proteins which are important in viral DNA replication, virion assembly and cellular transformation, and capsid proteins VP1, 2, and 3, which are structural-coating proteins. T-antigen proteins are subdivided into Large, Small, and sometimes Middle T-antigen proteins. T-antigen genes are expressed early and genes that encode capsid proteins are expressed late.

Shuda et a ${ }^{40}$ described multiple distinct LT antigen mutations in the MCPyV of MCC tumors. Normal LT antigen protein contains binding sites for retinoblastoma tumor suppressor protein and heat shock protein-tumor suppressor and cell cycle regulatory proteins and a helicase domainnecessary for autonomous viral replication. The mutations found by Shuda et al resulted in stop codons within the LT antigen transcript and subsequent truncation (shortening) of 


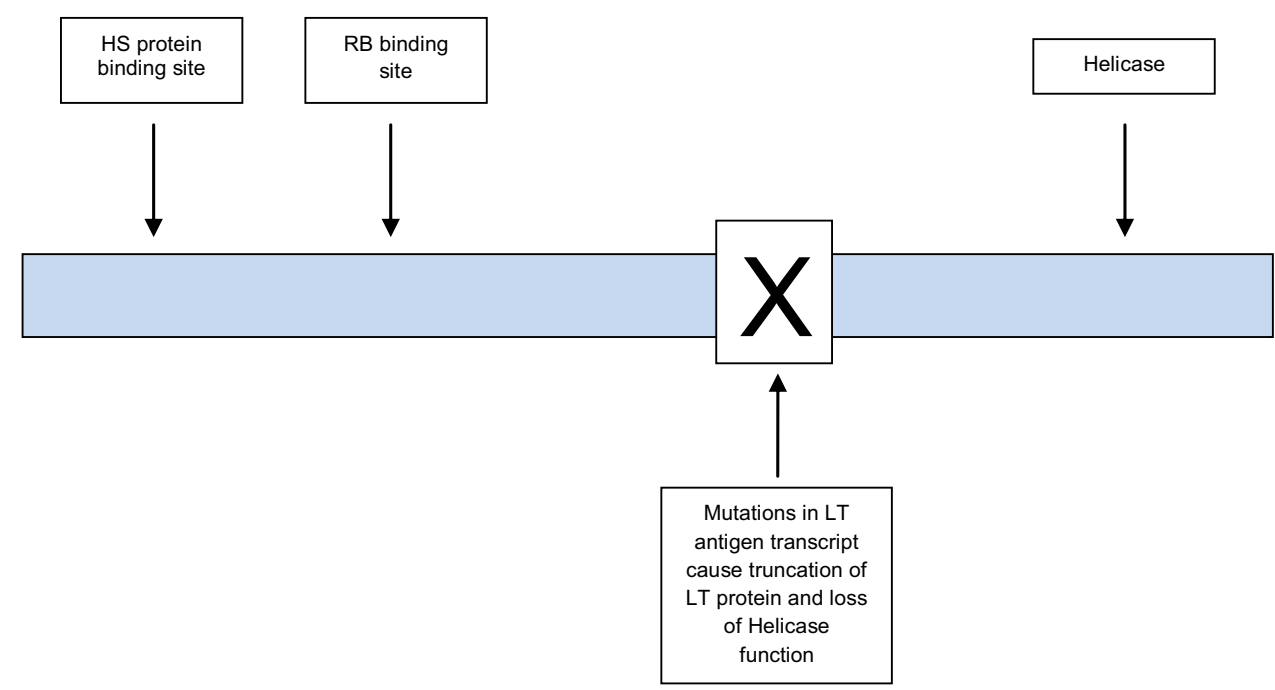

Figure 5 LT antigen protein. LT, large T antigen; HS, heat shock; RB, retinoblastoma tumor suppressor protein.

the LT antigen protein. Truncation of the LT antigen protein leads to loss of the virus's ability to replicate epigenomically, implying integration before clonal expansion. In this study, while multiple distinct mutations in the LT antigen transcript were found, all mutations led to loss of helicase function but preservation of retinoblastoma tumor suppressor protein and heat shock protein binding sites (Fig. 5). It is interesting to note that no CC $\rightarrow$ TT signature mutations for ultravioletinduced changes were found among the LT antigen mutations.

Since its discovery, MCPyV has been found in normal skin samples from both immune competent and immunocompromised patients and in a minority of BCC, invasive SCC, and SCC in situ. However, no connection between MCPyV and these tumors has yet been drawn. ${ }^{41-43}$

Other investigators have shown that nearly $90 \%$ of healthy non-MCC adults possess antibodies to the MCPyVs. As with other polyoma viruses, exposure to MCPyV likely occurs in childhood. ${ }^{44,45}$ Although patients with MCC have higher titers of antibodies to MCPyV VPl capsid proteins compared with control subjects, these titers have not been shown to correlate with clinical course and appear to offer no protection against MCC. Recently, titers of antibodies recognizing MCPyV T antigens were found to fall in treated MCC patients and to rise preceding the detection of metastases. ${ }^{46}$

Since the discovery of the MCC polyoma virus, additional studies have substantiated that approximately $80 \%$ of MCC tumors contain MCPyV. In one Australian study, however, $\mathrm{MCPyV}$ was found in a significantly lower percentage of MCC tumors. ${ }^{47}$ These results may imply MCPyV's association in only a subset of MCCs. It is interesting to note that MCC cell lines that have monoclonally integrated $\mathrm{MCPyV}$ genomes form loose suspensions in culture, whereas MCC cell lines without MCPyV grow as tightly adherent spindle shaped cells. ${ }^{48}$

Recently, MCPyV has been found in highly purified leukemic cells of approximately one-quarter of CLL patients studied. A novel LT antigen mutation was found in a subset of these MCPyV-positive CLL patients that was not seen in the $\mathrm{MCPyV}$ of normal controls. This discovery may argue for a potential role for $\mathrm{MCPyV}$ in a subset of CLL cases. ${ }^{49}$

Discovery of alterations in the LT antigen in the MCPyV has led to development of a monoclonal antibody against it, CM2B4. This antibody has been demonstrated to be useful in both formalin-fixed paraffin embedded and fresh, frozen specimens and has been used to investigate the presence of $\mathrm{MCPyV}$ in these samples. It stains positively in approximately $70 \%$ of MCCs and may prove to be a useful adjunct to CK20 in the identification of MCC tumor cells. ${ }^{5}$

\section{Conclusions}

MCC is an aggressive cutaneous malignancy whose diagnosis is often overlooked at the time of clinical presentation. The incidence of MCC is on the rise, and increasing awareness along with advances in immunohistopathologic staining have greatly aided in diagnosis. New staging recommendations, practice guidelines, and diagnostic coding are of considerable benefit to patient care, and a multidisciplinary approach is vital to optimize outcomes. The emerging role of $\mathrm{MCPyV}$ in MCC provides exciting insights into the etiology of this rare tumor.

\section{References}

1. Hodgson NC: Merkel cell carcinoma: Changing incidence trends. J Surg Oncol 89:1-4, 2005

2. Lemos B, Nghiem P: Merkel cell carcinoma: More deaths but still no pathway to blame. J Invest Dermatol 127:2100-2103, 2007

3. Hitchcock CL, Bland KI, Laney RG 3rd: Neuroendocrine (Merkel cell) carcinoma of the skin. Its natural history, diagnosis, and treatment. Ann Surg 207:201-207, 1988

4. Engels EA, Frisch M, Goedert JJ, et al: Merkel cell carcinoma and HIV infection. Lancet 359:497-498, 2002

5. Busam KJ, Jungbluth AA, Rekthman N, et al: Merkel cell polyomavirus expression in Merkel cell carcinomas and its absence in combined tumors and pulmonary neuroendocrine carcinomas. Am J Surg Pathol 3:1378-1385, 2009 
6. Tadmor T, Aviv A, Polliack A: Merkel cell carcinoma, chronic lymphocytic leukemia and other lymphoproliferative disorders: An old bond with possible new viral ties. Ann Oncol 22:250-256, 2011

7. Lemos BD, Storer BE, Iyer JG, et al: Pathologic nodal evaluation improves prognostic accuracy in Merkel cell carcinoma: Analysis of 5823 cases as the basis of the first consensus staging system. J Am Acad Dermatol 63:751-761, 2010

8. Halata Z, Grim M, Bauman KI: Friedrich Sigmund Merkel and his "Merkel cell," morphology, development, and physiology: Review and new results. Anat Rec A Discov Mol Cell Evol Biol 271:225-239, 2003

9. Toker C: Trabecular carcinoma of the skin. Arch Dermatol 105:107110,1972

10. Heath M, Jaimes N, Lemos B, et al: Clinical characteristics of Merkel cell carcinoma at diagnosis in 195 patients: The AEIOU features. J Am Acad Dermatol 58:375-381, 2008

11. Moll I, Roessler M, Brandner JM, et al: Human Merkel cells—Aspects of cell biology, distribution and functions. Eur J Cell Biol 84:259-271, 2005

12. Smith KR Jr: The Haarscheibe. J Invest Dermatol 69:68-74, 1977

13. Eispert AC, Fuchs F, Brandner JM, et al: Evidence for distinct populations of human Merkel cells. Histochem Cell Biol 132:83-93, 2009

14. Van Keymeulen A, Mascre G, Youseff KK, et al: Epidermal progenitors give rise to Merkel cells during embryonic development and adult homeostasis. J Cell Biol 187:91-100, 2009

15. Maricich SM, Wellnitz SA, Nelson AM, et al: Merkel cells are essential for light-touch responses. Science 324:1580-1582, 2009

16. Moll R, Löwe A, Laufer J: Cytokeratin 20 in human carcinomas. A new histodiagnostic marker detected by monoclonal antibodies. Am J Pathol 140:427-447, 1992

17. Moll I, Kuhn C, Moll R: Cytokeratin 20 is a general marker of cutaneous Merkel cells while certain neuronal proteins are absent. J Invest Dermatol 104:910-915, 1995

18. Calder KB, Smoller BR: New insights into Merkel cell carcinoma. Adv Anat Pathol 17:155-161, 2010

19. Walsh NM: Primary neuroendocrine (Merkel cell) carcinoma of the skin: Morphologic diversity and implications thereof. Hum Pathol 32 680-689, 2001

20. Becker JC: Merkel cell carcinoma. Ann Oncol 21 suppl 7:vii81-vii85, 2010

21. Bobos M, Hytiroglou P, Kostopoulos I, et al: Immunohistochemical distinction between Merkel cell carcinoma and small cell carcinoma of the lung. Am J Dermatopathol 28:99-104, 2006

22. McCalmont TH: Paranuclear dots of neurofilament reliably identify Merkel cell carcinoma. J Cutan Pathol 37:821-823, 2010

23. Rao P, Balzer BL, Lemos BD, et al: Protocol for the examination of specimens from patients with Merkel cell carcinoma of the skin. Arch Pathol Lab Med 134:341-344, 2010

24. Gupta SG, Wang LC, Peñas PF, et al: Sentinel lymph node biopsy for evaluation and treatment of patients with Merkel cell carcinoma: The Dana Farber experience and meta-analysis of the literature. Arch Dermatol 142:685-690, 2006

25. Balch CM, Soong SJ, Gershenwald JE, et al: Prognostic factors analysis of 17,600 melanoma patients: Validation of the American Joint Committee on Cancer melanoma staging system. J Clin Oncol 19:36223634,2001

26. Beebe M, Dalton JA, Espronceda M, et al: CPT 2009 Professional Edition. Chicago, American Medical Association, 2009

27. Miller SJ, Alam M, Andersen J, et al: Merkel cell carcinoma. J Natl Compr Cancer Netw 7:322-332, 2009
28. Bichakjian CK, Lowe L, Lao CD, et al: Merkel cell carcinoma: Critical review with guidelines for multidisciplinary management. Cancer 110: $1-12,2007$

29. Mortier L, Mirabel X, Fournier C, et al: Radiotherapy alone for primary Merkel cell carcinoma. Arch Dermatol 139:1587-1590, 2003

30. Veness M, Foote M, Gebski V, et al: The role of radiotherapy alone in patients with Merkel cell carcinoma: Reporting the Australian experience of 43 patients. Int J Radiat Oncol Biol Phys 78:703-709, 2010

31. Stokes JB, Graw KS, Dengel LT, et al: Patients with Merkel cell carcinoma tumors $<$ or $=1.0 \mathrm{~cm}$ in diameter are unlikely to harbor regional lymph node metastasis. J Clin Oncol 27:3772-3777, 2009

32. Allen PJ, Bowne WB, Jaques DP, et al: Merkel cell carcinoma: Prognosis and treatment of patients from a single institution. J Clin Oncol 23: 2300-2309, 2005

33. Lewis KG, Weinstock MA, Weaver AL, et al: Adjuvant local irradiation for Merkel cell carcinoma. Arch Dermatol 142:693-700, 2006

34. Mojica P, Smith D, Ellenhorn JD: Adjuvant radiation therapy is associated with improved survival in Merkel cell carcinoma of the skin. J Clin Oncol 25:1043-1047, 2007

35. Fang LC, Lemos B, Douglas J, et al: Radiation monotherapy as regional treatment for lymph node-positive Merkel cell carcinoma. Cancer 116: 1783-1790, 2010

36. Bichakjian CK, Coit DG, Wong SL: Radiation versus resection for Merkel cell carcinoma. Cancer 116:1620-1622, 2010

37. Voog E, Biron P, Martin JP, et al: Chemotherapy for patients with locally advanced or metastatic Merkel cell carcinoma. Cancer 85:2589-2595, 1999

38. Garneski KM, Nghiem P: Merkel cell carcinoma adjuvant therapy: Current data support radiation but not chemotherapy. J Am Acad Dermatol 57:166-169, 2007

39. Feng H, Shuda M, Chang Y, et al: Clonal integration of a polyomavirus in human Merkel cell carcinoma. Science 319:1096-1100, 2008

40. Shuda M, Feng H, Kwun HJ, et al: T antigen mutations are a human tumor-specific signature for Merkel cell polyomavirus. Proc Natl Acad Sci U S A 105:16272-16277, 2008

41. Wieland U, Mauch C, Kreuter A, et al: Merkel cell polyomavirus DNA in persons without Merkel cell carcinoma. Emerg Infect Dis 15:14961498, 2009

42. Kassem A, Technau K, Kurz AK, et al: Merkel cell polyomavirus sequences are frequently detected in nonmelanoma skin cancer of immunosuppressed patients. Int J Cancer 125:356-361, 2009

43. Dworkin AM, Tseng SY, Allain DC, et al: Merkel cell polyomavirus in cutaneous squamous cell carcinoma of immunocompetent individuals. J Invest Dermatol 129:2868-2874, 2009

44. Pastrana DV, Tolstov YL, Becker JC, et al: Quantitation of human seroresponsiveness to Merkel cell polyomavirus. PLoS Pathog 5:e1000578, 2009

45. Kean JM, Rao S, Wang M, et al: Seroepidemiology of human polyomaviruses. PLoS Pathog 5 el000363, 2009

46. Paulson KG, Carter JJ, Johnson LG, et al: Antibodies to Merkel cell polyomavirus $\mathrm{T}$ antigen oncoproteins reflect tumor burden in Merkel cell carcinoma patients. Cancer Res 70:8388-8397, 2010

47. Garneski KM, Warcola AH, Feng Q, et al: Merkel cell polyomavirus is more frequently present in North American than Australian Merkel cell carcinoma tumors. J Invest Dermatol 129:246-248, 2009

48. Shuda M, Arora R, Kwun HJ, et al: Human Merkel cell polyomavirus infection I. MCV T antigen expression in Merkel cell carcinoma, lymphoid tissues and lymphoid tumors. Int J Cancer 125:1243-1249, 2009

49. Pantulu ND, Pallasch CP, Kurz AK, et al: Detection of a novel truncating Merkel cell polyomavirus large T antigen deletion in chronic lymphocytic leukemia cells. Blood 116:5280-5284, 2010 